\title{
A HYPERGEOMETRIC INEQUALITY
}

\author{
ATUL DIXIT, VICTOR H. MOLL, AND VERONIKA PILLWEIN
}

\begin{abstract}
A sequence of coefficients that appeared in the evaluation of a rational integral has been shown to be unimodal. The original proof is based on a inequality for hypergeometric functions. A generalization is presented.
\end{abstract}

\section{INTRODUCTION}

A sequence of numbers $\left\{a_{k}: 0 \leq k \leq n\right\}$ is called unimodal if there is an index $k^{*}$ such that $a_{k-1} \leq a_{k}$ for $1 \leq k \leq k^{*}$ and $a_{k-1} \geq a_{k}$ for $k^{*}+1 \leq k \leq n$. The prototypical example of unimodal sequences is $a_{k}=\left(\begin{array}{l}n \\ k\end{array}\right)$. A polynomial $P(x)$ is called unimodal if its sequence of coefficients is unimodal.

A simple criteria for unimodality of a polynomial was established in [4]:

Theorem 1.1. If $P(x)$ is a polynomial with positive nondecreasing coefficients, then $P(x+1)$ is unimodal.

The original motivation for this result was the question of unimodality of the polynomial

$$
P_{m}(a)=\sum_{\ell=0}^{m} d_{\ell}(m) a^{\ell}
$$

with

$$
d_{\ell}(m)=2^{-2 m} \sum_{k=\ell}^{m} 2^{k}\left(\begin{array}{c}
2 m-2 k \\
m-k
\end{array}\right)\left(\begin{array}{c}
m+k \\
m
\end{array}\right)\left(\begin{array}{l}
k \\
\ell
\end{array}\right) .
$$

This example appeared in the evaluation of the formula

$$
\int_{0}^{\infty} \frac{d x}{\left(x^{4}+2 a x^{2}+1\right)^{m+1}}=\frac{\pi}{2} \frac{P_{m}(a)}{[2(a+1)]^{m+1 / 2}}
$$

given in [5]. A variety of proofs of (1.3) can be found in [3] and properties of the coefficients $\left\{d_{\ell}(m)\right\}$ have been reviewed in [26].

A property stronger than unimodality is that of logconcavity: a sequence of positive numbers $\left\{a_{k}: 0 \leq k \leq n\right\}$ is called logconcave if $a_{k}^{2} \geq a_{k-1} a_{k+1}$ for $1 \leq k \leq n-1$. As before, a polynomial is called logconcave if its sequence of coefficients is logconcave. The logconcavity of $P_{m}(a)$ was first established by M. Kauers and P. Paule in [23] using computer algebra, in particular algorithms for automatically deriving recurrences for multiple sums and Cylindrical Algebraic Decomposition (CAD), and by W. Y. Chen et al in $[10,11,12,13]$ by a variety of classical techniques.

Date: March 18, 2014.

2010 Mathematics Subject Classification. Primary 33C05.

Key words and phrases. Hypergeometric function, unimodal polynomials, monotonicity. 
The original proof of unimodality for $P_{m}(a)$ in [6] was based on the monotonicity of

$$
T(m)=\sum_{r=2}^{m+1}\left(\begin{array}{c}
2 r \\
r
\end{array}\right)\left(\begin{array}{c}
m+1 \\
r
\end{array}\right) \frac{(r-1)}{2^{r}\left(\begin{array}{c}
4 m \\
r
\end{array}\right)} .
$$

This proof was revisited in [2] and the hypergeometric representation

$$
T(m)=1-{ }_{1} F_{2}\left(\begin{array}{c}
\frac{1}{2},-1-m \\
-4 m
\end{array} \mid 2\right)+\frac{m+1}{4 m}{ }_{1} F_{2}\left(\begin{array}{c}
\frac{3}{2},-m \\
1-4 m
\end{array} \mid 2\right)
$$

was used to give a new proof of the monotonicity of $T(m)$ and also to establish the value $\lim _{m \rightarrow \infty} T(m)=2-\sqrt{2}$.

The monotonicity of $T(m)$ yields a curious inequality, that is transformed via contiguous identities for hypergeometric functions to the form

$$
\begin{aligned}
{ }_{2} F_{1}\left(\begin{array}{c}
\frac{3}{2},-m-2 \\
-4 m-4
\end{array} \mid 2\right)-{ }_{2} F_{1}\left(\begin{array}{c}
\frac{3}{2}, \\
-m-1 \\
-4 m
\end{array} \mid 2\right)> \\
\quad 3\left[{ }_{2} F_{1}\left(\begin{array}{c}
\frac{1}{2},-m-2 \\
-4 m-4
\end{array} \mid 2\right)-{ }_{2} F_{1}\left(\begin{array}{c}
\frac{1}{2},-m-1 \\
-4 m
\end{array} \mid 2\right)\right]
\end{aligned}
$$

The goal of this note is to prove the generalization of the previous inequality conjectured in [2]. The main result is stated next.

Theorem 1.2. The inequality

$$
\begin{aligned}
{ }_{2} F_{1}\left(\begin{array}{c}
\frac{3}{2},-m-2 \\
-4 m-4
\end{array} \mid 4 x\right)-{ }_{2} F_{1} & \left(\begin{array}{c}
\frac{3}{2},-m-1 \\
-4 m
\end{array} \mid 4 x\right)> \\
& 3\left[{ }_{2} F_{1}\left(\begin{array}{c}
\frac{1}{2},-m-2 \\
-4 m-4
\end{array} \mid 4 x\right)-{ }_{2} F_{1}\left(\begin{array}{c}
\frac{1}{2},-m-1 \\
-4 m
\end{array} \mid 4 x\right)\right]
\end{aligned}
$$

holds for $x \geq \frac{1}{2}$.

\section{An AUtomatic PRoof}

This section provides an automatic proof of Theorem 1.2. The main result is first rewritten using

$$
\ell_{m}(x)={ }_{2} F_{1}\left(\begin{array}{c}
\frac{3}{2},-m-2 \\
-4 m-4
\end{array} \mid 4 x\right), \quad \text { and } \quad r_{m}(x)=3{ }_{2} F_{1}\left(\begin{array}{c}
\frac{1}{2},-m-2 \\
-4 m-4
\end{array} \mid 4 x\right),
$$

in the form

$$
\ell_{m}(x)-\ell_{m-1}(x)>r_{m}(x)-r_{m-1}(x) .
$$

The expansion of the terms in the previous inequality becomes nicer if written as

$$
L_{m}(x):=\ell_{m}(x)-r_{m}(x)>\ell_{m-1}(x)-r_{m-1}(x)=L_{m-1}(x) .
$$

Using any of the packages $[14,25]$ dealing with symbolic summation and holonomic closure properties that are available nowadays, it is easy to discover (and thus prove) a second order recurrence satisfied by $L_{m}(x)$. In theory, this would make this inequality accessible to the automatic inequality prover developed by S. Gerhold and M. Kauers $[18,20,21]$. These methods need as input only a defining recurrence and sufficiently many initial values. This method uses as an essential tool Cylindrical Algebraic Decomposition $[8,15,16]$ (CAD), which was introduced to solve the 
problem of quantifier elimination over the field of real numbers. Given a quantified logical formula of polynomial equalities and inequalities, it computes an equivalent, quantifier-free formula. If the given formula does not contain any free variables, then the output is one of the logical constants true or false. The input class can be generalized to rational or algebraic functions, since these kinds of functions can be expressed in terms of polynomial equalities. For a recent overview on how and when to apply CAD see [22].

The run-time and memory requirements of CAD computations depend doubly exponential (in the worst case) on the number of variables and the degrees of the appearing polynomials. For the inequality discussed here, the calculations did not terminate within a reasonable amount of time. The recurrence is linear and only of order two, but the polynomial coefficients have degree 16 in $m$ and degrees 9,10 in $x$. This might explain the lack of termination in a reasonable time. Besides the computational complexity, this may also be because the method of Gerhold and Kauers is not an algorithm in the strict sense, since termination is not guaranteed for an arbitrary input [24, 28]. Despite this fact, this procedure has been applied successfully in proving different non-trivial inequalities, e.g., on orthogonal polynomials $[1,19,27]$. For the inequality at hand the approach does not seem to succeed and hence a different line of computer-assisted proof that also exploits CAD has been chosen.

The series expansion of $L_{m}(x)$ can be computed as follows,

$$
\begin{aligned}
L_{m}(x) & =\sum_{k \geq 0}\left(\left(\frac{3}{2}\right)_{k}-3\left(\frac{1}{2}\right)_{k}\right) \frac{(-m-2)_{k}}{(-4 m-4)_{k} k !}(4 x)^{k} \\
& =-2+\sum_{k \geq 2} \frac{(-m-2)_{k}\left(\frac{1}{2}\right)_{k}}{(-4 m-4)_{k} k !}(k-1) 2^{2 k+1} x^{k}=-2+\sum_{k \geq 2} c(m, k) x^{k},
\end{aligned}
$$

with coefficients

$$
c(m, k)=\frac{(-m-2)_{k}\left(\frac{1}{2}\right)_{k}}{(-4 m-4)_{k} k !}(k-1) 2^{2 k+1} .
$$

Here $(a)_{k}=a(a+1) \cdots(a+k-1)$ denotes the Pochhammer symbol (or rising factorial). Note that the sums above are finite (and thus the $L_{m}(x)$ are polynomials) because of the factor $(-m-2)_{k}$ in the numerator.

It is easy to see that for $k \geq 2$ and any $m \geq 2$ these coefficients are nonnegative. The only negative contributions stem from the Pochhammer symbols in the numerator and denominator with the same number of factors. The coefficients $c(m, 1)$ vanish and the constant coefficients $c(m, 0)=-2$ for all $m$. Hence the forward difference considered here may be written as

$$
L_{m}(x)-L_{m-1}(x)=\sum_{k=2}^{m+1}\left(\frac{c(m, k)}{c(m-1, k)}-1\right) c(m-1, k) x^{k}+c(m, m+2) x^{m+2},
$$

with the shift quotient $\rho(m, k)=c(m, k) / c(m-1, k)$ given by

$$
\rho(m, k)=\frac{(m+2)(-k+4 m+1)(-k+4 m+2)(-k+4 m+3)(-k+4 m+4)}{8(m+1)(2 m+1)(4 m+1)(4 m+3)(-k+m+2)} .
$$

This shift quotient is a rational function, hence it is suitable as input for a CAD computation. Since $x \geq \frac{1}{2}$, and $c(m, m+2)>0$ for $m \geq 0$, it would be sufficient 
to prove that $\rho(m, k) \geq 1$ for all $m, k$. This is not the case, but for $k, m \geq 7$ CAD quickly shows that the shift quotient is bounded from below by one:

$\ln [1]:=$ Resolve[ForAll[ $\{m, k\}, m \geq 7 \& \& 7 \leq k \leq n+1, \rho[m, k] \geq 1],\{\}$, Reals] Out[1]= True

These simple observations gave rise to the idea to truncate $L_{m}(x)-L_{m-1}(x)$ at a fixed level that is provably positive and then to show that the tail of the expansion of $L_{m}(x)-L_{m-1}(x)$ is non-negative. All these steps can be carried out using CAD. The outline of the proof is as follows:

(1) Prove that $L_{m}(x)-L_{m-1}(x)>0$ for $m \leq 6$.

(2) Prove that the truncated version of the inequality with $k$ running from 2 to 6 is positive for all $m \geq 7$.

(3) Prove that the tails are non-negative.

The first cases for $m \leq 6$ are checked independently using CAD. Note that for $m=1$ the inequality is strict only for $x>\frac{1}{2}\left(L_{1}\left(\frac{1}{2}\right)=0\right)$. In the problem considered here, the Mathematica implementation of CAD [29] has been used, but certainly any other computer algebra system capable of executing CAD $[7,17,9]$ would be able to handle the given inequalities. In Mathematica the commands can be executed as follows (where $L[m, x]=L_{m}(x)$ ),

$\operatorname{In}[2]:=\operatorname{Resolve}\left[\right.$ For All $\left[x, x>\frac{1}{2}, L[1, x]-L[0, x]>0\right],\{\}$, Reals]

Out $[2]=$ True

$\ln [3]:=$ Table[Resolve[ForAll $\left[x, x \geq \frac{1}{2}, L[m, x]-L[m-1, x]>0\right],\{\}$, Reals],$\left.\{m, 2,6\}\right]$ Out $[3]=\{$ True, True, True, True, True $\}$

In the second step we consider the truncated power series that is a polynomial in $m$ and $x$ of fixed degree,

$$
\begin{aligned}
T_{m}(x)=-2 & +\sum_{k=2}^{6} c(m, k) x^{k} \\
=-2 & +\frac{3(m+2) x^{2}}{4 m+3}+\frac{10 m(m+2) x^{3}}{(2 m+1)(4 m+3)}+\frac{105(m-1) m(m+2) x^{4}}{2(2 m+1)(4 m+1)(4 m+3)} \\
& +\frac{63(m-2)(m-1)(m+2) x^{5}}{(2 m+1)(4 m+1)(4 m+3)}+\frac{1155(m-3)(m-2)(m-1)(m+2) x^{6}}{4(2 m+1)(4 m-1)(4 m+1)(4 m+3)} .
\end{aligned}
$$

The following CAD computation quickly verifies that $T_{m+1}(x)-T_{m}(x)>0$ holds for $m \geq 7$ and $x \geq \frac{1}{2}$,

$$
\left.\ln [4]==\text { Resolve[ForAll }\left[\{m, x\}, m \geq 7 \& \& x \geq \frac{1}{2}, T[m, x]-T[m-1, x]>0\right],\{\}, \text { Reals }\right]
$$
Out $[4]=$ True

The summary of the computations carried out so far is this: For $m \leq 7 \mathrm{CAD}$ shows that $L_{m}(x)>L_{m-1}(x)$. For $m \geq 7$ and $x \geq \frac{1}{2}$ we have,

$$
\begin{aligned}
L_{m}(x)-L_{m-1}(x)= & T_{m}(x)-T_{m-1}(x) \\
& +\sum_{k=7}^{m+1} \underbrace{(\rho(m, k)-1) c(m-1, k) x^{k}}_{\geq 0}+\underbrace{c(m+2, m+2) x^{m+2}}_{\geq 0} \\
\geq & T_{m}(x)-T_{m-1}(x)>0,
\end{aligned}
$$

where for the penultimate estimate we use that the shift quotient $\rho(m, k) \geq 1$ for $m, k \geq 7$ as shown above. This completes the proof. 


\section{A DIFFERENT APPROACH}

Using automated guessing it is possible to derive a second order ordinary differential equation satisfied by $y(x)=L_{m}(x)$ that (once found) is easy to check. This procedure is described here and is used to established the inequality in Theorem 1.2 for $x \geq 1$. This is not the optimal range, but the techniques employed here are simpler.

The equation obtained for $y(x)=L_{m}(x)$ is

$$
\begin{aligned}
x(3 x-2)(4 x-1) y^{\prime \prime}(x)-\left(12 m x^{2}-20 m x+8 m+6 x^{2}-11 x+8\right) y^{\prime}(x) & \\
& -6(m+2) x y(x)=0 .
\end{aligned}
$$

The same technique is then used to find (and subsequently prove) the mixed difference-differential relation

$$
d(m, x) L_{m+1}(x)=c_{1}(m, x) L_{m}^{\prime}(x)+c_{0}(m, x) L_{m}(x),
$$

with

$$
\begin{aligned}
d(m, x)= & 2(m+2)(2 m+3)(4 m+5)(4 m+7)\left(16 m^{2}-104 m+425\right) \cdot \\
& \cdot(1-4 x)^{2}(3 x-2)(6 x+7), \\
c_{1}(m, x)= & \left(16 m^{2}-104 m+425\right) x(6 x+7)\left(16 m^{3}\left(81 x^{4}-165 x^{3}+107 x^{2}-25 x+2\right)\right. \\
& +24 m^{2}\left(270 x^{4}-548 x^{3}+368 x^{2}-87 x+7\right)+m\left(10656 x^{4}-21585 x^{3}\right. \\
& \left.\left.+15052 x^{2}-3605 x+292\right)+3\left(1920 x^{4}-3889 x^{3}+2824 x^{2}-686 x+56\right)\right), \\
c_{0}(m, x)= & (m+2)\left(16 m^{2}-104 m+425\right)(6 x+7)\left(64 m^{3}(1-4 x)^{2}(3 x-2)\right. \\
& +24 m^{2}\left(27 x^{4}+566 x^{3}-671 x^{2}+228 x-24\right) \\
& +m\left(1944 x^{4}+19953 x^{3}-23920 x^{2}+8132 x-856\right) \\
& \left.+3\left(480 x^{4}+3249 x^{3}-3914 x^{2}+1330 x-140\right)\right) .
\end{aligned}
$$

Using CAD and these equations it is possible to show that $T_{m}(x) \geq 0$ for $m \geq 3$ and $x \geq 1$, and that $L_{m}(x) \geq 0$ for $m \leq 7$ and $x \geq 1$. Since

$$
L_{m}(x)=T_{m}(x)+\sum_{k \geq 7} c(m, k) x^{k}
$$

with non-negative coefficients $c(m, k)$, it follows that $L_{m}(x) \geq 0$ for all $x \geq 1$. The coefficients of $L_{m}^{\prime}(x)$ are all non-negative (the constant coefficient -2 vanishes), and hence also $L_{m}^{\prime}(x) \geq 0$ for all $m, k \geq 2$ and $x>0$. The relation (3.1) then yields

$$
L_{m+1}(x)-L_{m}(x)=\frac{c_{1}(m, x)}{d(m, x)} L_{m}^{\prime}(x)+\frac{c_{0}(m, x)-d(m, x)}{d(m, x)} L_{m}(x) .
$$

A routine use of $\mathrm{CAD}$, shows the rational function coefficients on the right hand side above are positive for $x \geq 1$. This completes the proof.

Acknowledgments. The second author acknowledges the partial support of NSFDMS 1112656. The first author is a post-doctoral fellow, funded in part by the same grant. The third named author was supported by the Austrian Science Fund (FWF) under grant P22748-N18. 


\section{REFERENCES}

[1] H. Alzer, S. Gerhold, M. Kauers, and A. Lupas. On Turan's Inequality for Legendre Polynomials. Expositiones Mathematicae, 25(2):181-186, May 2007.

[2] T Amdeberhan, A Dixit, X. Guan, L. Jiu, and V. Moll. The unimodality of a polynomial coming from a rational integral. back to the original proof. Submitted for publication, 2013.

[3] T. Amdeberhan and V. Moll. A formula for a quartic integral: a survey of old proofs and some new ones. The Ramanujan Journal, 18:91-102, 2009.

[4] G. Boros and V. Moll. A criterion for unimodality. Elec. Jour. Comb., 6:1-6, 1999.

[5] G. Boros and V. Moll. An integral hidden in Gradshteyn and Ryzhik. Jour. Comp. Applied Math., 106:361-368, 1999.

[6] G. Boros and V. Moll. A sequence of unimodal polynomials. Jour. Math. Anal. Appl., 237:272-287, 1999.

[7] C.W. Brown. QEPCAD B - a program for computing with semi-algebraic sets. Sigsam Bulletin, 37(4):97-108, 2003.

[8] B. Caviness and J. R. Johnson. Quantifier elimination and cylindrical algebraic decomposition. Texts and Monographs in Symbolic Computation. Springer-Verlag, 1st edition, 1998.

[9] C. Chen, M. Moreno Maza, B. Xia, and L. Yang. Computing cylindrical algebraic decomposition via triangular decomposition. In Proceedings of ISSAC'09, pages 95-102, New York, NY, USA, 2009. ACM.

[10] W. Y. C. Chen and C. C. Y. Gu. The reverse ultra log-concavity of the Boros-Moll polynomials. Proc. Amer. Math. Soc., 137:3991-3998, 2009.

[11] W. Y. C. Chen and E. X. W. Xia. The ratio monotonicity of the Boros-Moll polynomials. Math. Comp., 78:2269-2282, 2009.

[12] W. Y. C. Chen and E. X. W. Xia. 2-log-concavity of the Boros-Moll polynomials. Preprint, 2011.

[13] W. Y. C. Chen and E. X. W. Xia. A proof of Moll's minimum conjecture. European J. Combin., 34:787-791, 2013.

[14] F. Chyzak. Fonctions holonomes en calcul formel. Thèse universitaire, École polytechnique, 1998. INRIA, TU 0531. 227 pages.

[15] G. E. Collins. Quantifier elimination for the elementary theory of real closed fields by cylindrical algebraic decomposition. Lecture Notes in Computer Science, 33:134-183, 1975.

[16] G.E. Collins and H. Hong. Partial cylindrical algebraic decomposition for quantifier elimination. Journal of Symbolic Computation, 12(3):299-328, 1991.

[17] A. Dolzmann and T. Sturm. Redlog: computer algebra meets computer logic. Sigsam Bulletin, 31(2):2-9, 1997.

[18] S. Gerhold and M. Kauers. A procedure for proving special function inequalities involving a discrete parameter. In M. Kauers, editor, Proceedings of ISSAC'05, pages 156-162, 2005.

[19] S. Gerhold and M. Kauers. A computer proof of Turán's inequality. Journal of Inequalities in Pure and Applied Mathematics, 7(2):\#42, 2006.

[20] M. Kauers. SumCracker: A package for manipulating symbolic sums and related objects. Journal of Symbolic Computation, 41(9):1039-1057, 2006.

[21] M. Kauers. Computer algebra and special function inequalities. In Tewodros Amdeberhan and Victor H. Moll, editors, Tapas in Experimental Mathematics, volume 457 of Contemporary Mathematics, pages 215-235. AMS, 2008.

[22] M. Kauers. How To Use Cylindrical Algebraic Decomposition. Seminaire Lotharingien de Combinatoire, 65(B65a):1-16, 2011.

[23] M. Kauers and P. Paule. A computer proof of Moll's log-concavity conjecture. Proc. Amer. Math. Soc., 135:3837-3846, 2007.

[24] M. Kauers and V. Pillwein. When can we detect that a P-finite sequence is positive? In Stephen Watt, editor, Proceedings of ISSAC'10, pages 195-202, 2010.

[25] C. Koutschan. HolonomicFunctions (User's Guide). Technical Report 10-01, RISC Report Series, University of Linz, Austria, January 2010.

[26] D. Manna and V. Moll. A remarkable sequence of integers. Expositiones Mathematicae, 27:289-312, 2009.

[27] V. Pillwein. Positivity of certain sums over Jacobi kernel polynomials. Advances in Applied Mathematics, 41(3):365-377, 2008. 
[28] V. Pillwein. Termination Conditions for Positivity Proving Procedures. In M. Kauers, editor, Proceedings of ISSAC'13, pages 315-321, 2013.

[29] A. Strzeboński. Solving systems of strict polynomial inequalities. Journal of Symbolic Computation, 29:471-480, 2000.

Department of Mathematics, Tulane University, New Orleans, LA 70118

E-mail address: adixit@tulane.edu

Department of Mathematics, Tulane University, New Orleans, LA 70118

E-mail address: vhm@tulane.edu

Research Institute for Symbolic Computation, Johannes Kepler University, A-4040 Linz, Austria

E-mail address: veronika.pillwein@risc.jku.at 